\title{
Benign familial disease with muscle mounding and rippling
}

\author{
R J Burns, A H Bretag, P C Blumbergs, M G Harbord
}

\begin{abstract}
Four members of a family in three generations exhibited unusual clinical features of localised transient swelling of muscle induced by percussion (muscle mounding or myoedema) and were able, voluntarily, to induce rhythmic waves of contraction in certain muscles (muscle rippling or rolling). All had raised serum creatine kinase activity. Muscle biopsy in two members showed no specific abnormality. Experimental studies performed on excised intercostal muscle showed that abnormal "after-contractions" and increased sarcolemmal excitability could be demonstrated in vitro.
\end{abstract}

(F Neurol Neurosurg Psychiatry 1994;57:344-347)

Department of Neurology, Flinders Medical Centre, South Australia, Australia R J Burns

School of Pharmacy, University of South Australia, Australia A H Bretag

Institute of Medical and Veterinary Science, Australia P C Blumbergs

Department of Paediatrics, Flinders Medical Centre, South Australia, Australia M G Harbord

Correspondence to: Dr R J Burns, Departmen of Medicine, Flinders Medical Centre, Bedford Park 5042, South Australia, Australia.

Received 28 September 1992 and in final revise form 4 May 1993.

Accepted 7 May 1993
A remarkable muscle response that has seldom been associated with underlying myopathy is a propensity for skeletal muscle to mound or swell with local stimulation, sometimes called myoedema. It has mainly been noted as a curiosity, and may be seen in normal states. ${ }^{12}$ Little has been documented of an even more unusual condition known as muscle rippling or rolling in which wave-like self-propagating muscle contractions can be voluntarily induced. A predominantly familial disorder with prominent muscle mounding and muscle rippling has been described, ${ }^{3-8}$ but no investigations of in vitro muscle contractility have been published.

We report a family (father, two sons, and a grandson) with muscle mounding and muscle rippling. Clinical features are presented and the results of muscle biopsy and in vitro experimental studies of excised muscle on one member are described in detail.

\section{Case reports}

Case 1

A 19-year-old man was first seen in 1980. For two to three years he had noticed a rippling of his muscles that he could induce himself especially after a period of rest, in the cold, or in the early morning. He had also been aware that whenever his muscles were struck or knocked a localised, painless swelling would appear at the site of impact, subsiding within five to 10 seconds. He had occasional aching and stiffness of his muscles. Examination revealed a healthy appearing man with well developed muscles. There was no wasting, no fasciculations were seen, and no weakness could be demonstrated. His deep tendon reflexes were intact and symmetrical. A painless swelling appeared with light percussion of most muscles especially those around the shoulder. This conformed to the shape of the percussion hammer and lasted for about five to 10 seconds before subsiding (fig 1). It could be reproduced although some fatiguability was noted, and it occurred during prolonged rest and immediately after exercise. The patient was also able to demonstrate rippling or rolling of his muscles, especially in the pectoralis major, where it could be induced voluntarily. This consisted of a wave of rhythmic contractions in rapid succession, not associated with cramp or pain. Similar rippling could be initiated in response to pressure applied to the quadriceps by the examiner's hand.

Routine haematology, electrolytes, liver function, and thyroid function tests were normal. His serum creatine kinase was persistently raised varying from 250 to 530 $\mathrm{IU} / 1$ (normal <140 IU/1). An ischaemic exercise test gave a normal rise in lactate and pyruvate. EMG studies showed no resting
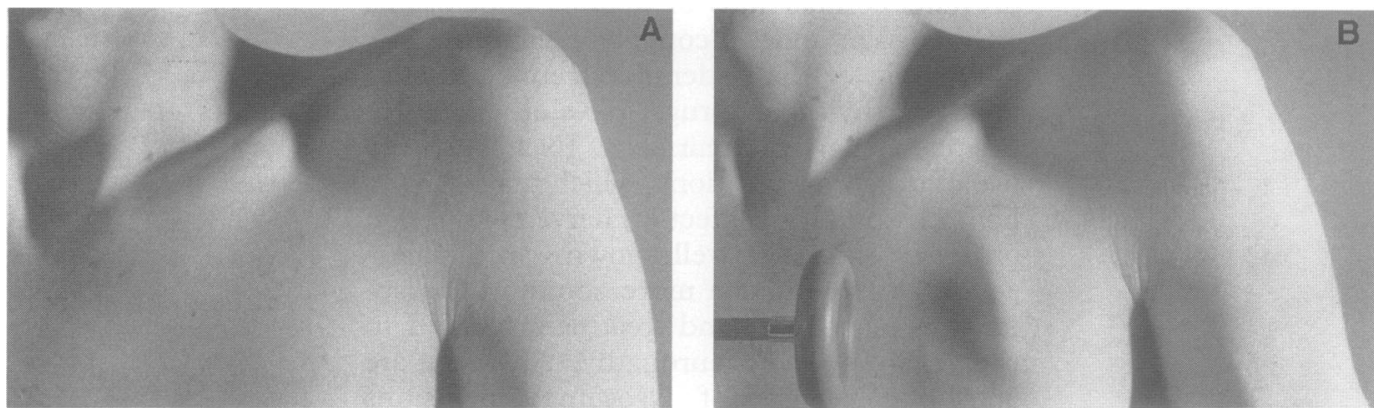

Figure 1 Muscle mounding in pectoral muscles of the 19-year-old proband, case 1. The photographs show the upper left anterior thoracic region before $(A)$ and after $(B)$ a brisk tap to the pectoral muscles with the tendon hammer. The mounding conforms to the shape of the tendon hammer and does not spread longitudinally with respect to the muscle fibres of the pectoralis major. 
activity and in particular, there were no myotonic discharges. The motor units were normal, with no evidence of a myopathic or neuropathic disorder. The muscle mounds were sampled and found to be electrically silent. A muscle biopsy was taken from the left vastus medialis. Light microscopy showed mild variation in muscle fibre size with occasional random small fibres. Routine morphometric studies in $\mathrm{pH} 9 \cdot 4,4 \cdot 6$, and $4 \cdot 2$ ATPase preparations showed selective type I fibre atrophy. The oxidative enzyme stains showed a normal intermyofibrillar network pattern. Myophosphorylase, periodic acidSchiff, oil-red-O, and Gomori trichrome $\mathrm{pH}$ 3.4 reactions were normal. Electron microscopy was normal. An intercostal muscle biopsy was also taken to perform specific physiological studies on the muscle. These will be described elsewhere.

In the 12 years since he was first seen he has remained well without change in his symptoms or signs.

\section{Case 2}

A 22-year-old man, the brother of case 1, had been aware of muscle mounding and rippling for about five years when first examined in 1980. The only signs were those of muscle mounding induced by percussion and he was able to make his muscles ripple. Serum creatine kinase was $774 \mathrm{IU} / 1$. An EMG was normal and the muscle mounds were electrically silent.

\section{Case 3}

The 47-year-old father of cases 1 and 2 had been able to make his muscles ripple since his teenage years and his muscles would mound when bumped or knocked. He complained of a tight sensation in his muscles with exercise but this in no way incapacitated him. His condition had not changed over the years. Mounding with percussion was seen in many muscles and he could induce muscle rippling voluntarily. His serum creatine kinase was $380 \mathrm{IU} / 1$.

\section{Case 4}

The eldest child in the third generation of this family had similar clinical findings. He was the son of case 2 and was examined in 1991 at nine years of age after a history of muscle aches during exercise and muscle swelling after minor trauma. Thigh muscle rippling, which occurred when he sat down after a prolonged period of standing, was also described.

Muscle mounding was apparent with percussion. His serum creatine kinase was 2317 IU/1 (normal <250 IU/1), whereas plasma lactate, total carnitine and free carnitine were normal. An EMG was normal. A needle muscle biopsy of the quadriceps revealed $12 \%$ Type IIC fibres but no evidence of fibre necrosis, inflammation, or fibrosis.

There was no history of consanguinity or malignant hyperthermia in the family.
Electrophysiological investigations in vitro An external intercostal muscle biopsy was obtained from the right sixth intercostal interspace approximately in the mid-axillary line from case 1 . The biopsy was maintained in synthetic interstitial fluid ${ }^{9}$ equilibrated with $95 \% \mathrm{O}_{2}, 5 \% \mathrm{CO}_{2}$ at room temperature during transport and dissection into usable bundles. Each preparation consisted of several hundred muscle fibres running parallel from tendon to tendon.

Muscle contraction was studied both isotonically and isometrically with Harvard No 356 and Grass FTO3 transducers coupled to Rikadenki and Beckman Dynograph penwriters respectively. Stimulation was via platinum wire electrodes placed close to the muscle. Normal stimulation comprised just supramaximal pulses of $1 \mathrm{~ms}$ duration in trains of three at $225 \mathrm{~Hz}$. Generally stimuli were applied once every 10 minutes to avoid the possibility of warm up phenomena.

Electrical properties were determined separately by conventional microelectrode techniques. Voltage recording electrodes contained potassium chloride $(3 \mathrm{~mol} / \mathrm{l})$ at $\mathrm{pH} 2$ and current passing electrodes were filled with potassium citrate $(2 \mathrm{~mol} / \mathrm{l})$.

\section{CONTRACTION}

Muscle contraction and relaxation in all isometric records and most isotonic records were normal. On a few occasions in the isotonically contracting preparation a secondary contraction developed either during or immediately after a normal relaxation (fig 2 ). Such

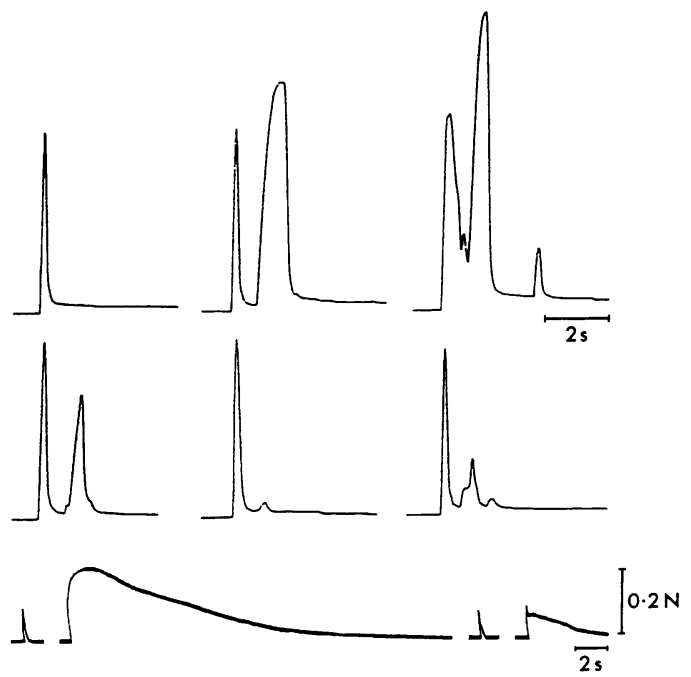

Figure 2 Contraction records of isolated external intercostal muscle from case 1 bathed in synthetic interstitial fluid at $37^{\circ} \mathrm{C}$. Top and middle rows are isotonic records showing an assortment of responses resulting from the standard stimulus of $3 \times 1$ ms just supramaximal pulses at $225 \mathrm{~Hz}$. The first record was the most common and seems to be completely normal. Occasionally the unusual and irregular secondary contractions shown in the other records occurred following or overlying the normal stimulated response. Isotonic muscle shortening was unscaled. Bottom row gives isometric records showing in sequence: a normal twitch in response to the standard stimulus, a myotonic contraction with the muscle bathed in low Cl synthetic interstitial fluid (Na diatrizoate substitution for $\mathrm{NaCl}$ ), another control twitch, and a myotonic contraction resulting from treatment with anthracene-9-carboxylate $\left(2 \cdot 25 \times 10^{-5} \mathrm{~mol} / \mathrm{l}\right)$ for 10 minutes before stimulation. The tension scale $(0 \cdot 2 \mathrm{~N})$ applies only to the isometric records. 
contractions did not occur spontaneously during the 10 minutes between stimuli. Systematic study of these "after-contractions" was precluded by their inconsistency. They disappeared over a period of an hour or two and did not seem to be provoked by either altered stimulation patterns or altered ionic composition of the bathing solution-for example, normal, double, or half normal $\mathrm{Ca}^{2+}$ or $\mathrm{K}^{+}$concentrations. By contrast low chloride solution readily and predictably induced quite different myotonic contractions (fig 2) as did chloride channel blockers such as anthracene-9-carboxylate $\left(2.25 \times 10^{-5}\right.$ $\mathrm{mol} / \mathrm{l}$; fig 2$)$ or furosemide $\left(1 \times 10^{-3} \mathrm{~mol} / \mathrm{l}\right.$; not shown). These myotonic responses were not different from those induced in normal rat muscle by the same agents. ${ }^{10}$

\section{ELECTRICAL PROPERTIES}

Resting potentials occurred in two ranges in surface fibres, $60-70 \mathrm{mV}$ and $80-90 \mathrm{mV}$. The first group tended to be electrically unexcitable and the second readily excitable. It is likely that the cells with low resting potential were damaged during dissection as deeper cells almost invariably had the higher resting potentials. As action potentials could only be initiated after insertion of a second electrode and this was only feasible in surface fibres, the low resting potential cells were ignored.

Cells producing action potentials had the high input resistance and consequently the low threshold currents $(<50 \mathrm{nA})$ typically found in normal human external intercostal fibres bathed in synthetic interstitial fluid. ${ }^{11}$ Latencies $>30 \mathrm{~ms}$, which are several times longer than normal (about $10 \mathrm{~ms}$ ), were seen, however, and repetitive firing occurred freely (fig 3 ) in response to maintained constant current stimulation at low threshold $(<75$ $\mathrm{nA}$ ). Many of the fibres fired repetitively with less than a $10 \%$ increase in rheobasic stimulating current.

These latter results are very different from those of Kwiecinski et al ${ }^{11}$ in normal human external intercostal fibres but are somewhat reminiscent of such fibres from goats with hereditary myotonia. ${ }^{12}$ There was, however, no sign whatever of spontaneously occurring action potentials, action potentials initiated by electrode insertion, or after-discharge of action potentials following stimulated repeti-

Figure 3 Intracellular microelectrode recording of freely firing repetitive action potentials lower action potentials (lower
trace) in response to the constant current pulse shown in the upper trace. Note the long latency to the second action potential indicating a slow accommodation. The record is from isolated external intercostal muscle (case 1) bathed in synthetic interstitial fluid at $32^{\circ} \mathrm{C}$.

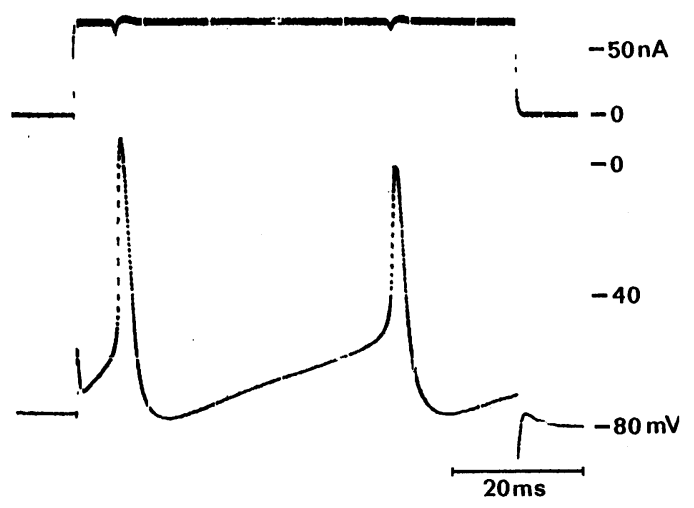

tive firing. Furthermore, resting potentials were usually very stable with no oscillatory tendencies. On firing action potentials, the muscle fibres contracted strongly and the electrodes often pulled out. Inhibitors of excitation-contraction-coupling such as hypertonic sucrose or dantrolene were not employed in case they interfered with the muscle abnormality. Pull out or deliberate withdrawal of an electrode from an excitable fibre was followed by microscopically obvious localised contracture of the fibre.

\section{Discussion}

The family described here bears a striking similarity to the family reported by Torbergsen $^{3}$ and the two families reported by Ricker et al. ${ }^{4}$ Aching or tightness in the leg muscles was complained of by all four families, but the muscle mounding was painless. The other features, including the absence of muscle weakness, raised creatine kinase, electrical silence, and non-specific changes only in the muscle biopsy are all consistent with this unique autosomal dominant inherited disease.

Although Torbergsen ${ }^{3}$ termed the condition "myotonia", he did not demonstrate myotonia electrically. Likewise, we did not find myotonia in our cases, nor have others. ${ }^{4-8}$ The stiffness and slowness seem to correlate with the rolling contractions produced by passive stretching of muscle immediately after contraction. Such mechanically activated contractions in antagonistic muscle groups would certainly slow and stiffen reciprocating movements. The abnormal involuntary eccentric muscular contractions resulting from this activity could also be responsible for the pain or aching and may well contribute to the raised creatine kinase. ${ }^{13}$

The rolling muscle contractions were shown to be electrically silent by us and others $^{378}$ although Hylton et $a l^{5}$ associated them with summated individual muscle fibre discharge varying in frequency and amplitude, a distinction that might separate their family from others. Although generally recorded as being voluntarily initiated, the wave-like contractions could sometimes be induced by prolonged compression of muscles, whereupon the spontaneous activity could be both seen and felt by the observer. ${ }^{7}$

Idiomuscular contraction of Schiff or myoedema has been known for 150 years. ${ }^{1}$ It can occur in healthy people but is seen particularly in severe debilitating disorders such as malnutrition, malabsorption, malignancy, and pulmonary tuberculosis as well as in hypothyroidism and the postictal state. ${ }^{121415}$ Under some circumstances the muscle mounding is accompanied by waves of self-propagating contraction that travel back and forth over the length of the muscle. ${ }^{1}$ Some studies have shown both the mounding and waves to be electrically silent ${ }^{12}$ and in this way distinct from myotonia. The precise physiological basis and reason for its pathophysiological accentuation are not known, but studies 
with normal ${ }^{16}$ and hypothyroid ${ }^{17}$ rat muscle indicate that it depends on release of $\mathrm{Ca}^{2+}$ from an intact sarcoplasmic reticulum. A functional abnormality of the sarcoplasmic reticulum could then be responsible for instances of exaggerated myoedema. ${ }^{16} 17$

Although the exact mechanism of the muscle mounding and muscle rippling has not been revealed by our clinical, histopathological, and experimental studies, several of our findings are unusual and informative. As far as we are aware there are no previous reports of the peculiar contraction-induced "after-contractions" seen transiently in the intercostal muscle in vitro. We suspect these to be the in vitro manifestation of the voluntarily induced muscle rippling or rolling. It seems that in each case they are precipitated by the immediately prior electrically induced muscle contractions and may be contingent on the passive stretch back towards normal resting length that follows active shortening in the isotonic recording apparatus. It should be noted that the maximum rate of shortening in the after-contraction is significantly less than during the electrically triggered contraction. We suggest that the after-contraction results from a secondary slow release of $\mathrm{Ca}^{2+}$ from the sarcoplasmic reticulum as a result of abnormal sensitivity, perhaps of the $\mathrm{Ca}^{2+}$ channels, to mechanical distortion.

The voluntarily induced rippling or rolling is propagated slowly enough to be quite visible and thus much more slowly than normal action potentials in muscles. It may be that the unusual mechanical sensitivity is itself responsible for the propagation of a mechanical wave of contraction or that there is slow propagation of an internal sarcoplasmic action potential. In either case, as myotonic electrical activity of the sarcolemma does not seem to be involved, the rippling or rolling muscle activity is probably not strictly an after-contraction but should more accurately be termed a propagating after-contracture.

The heightened electrical excitability of the sarcolemma, although not sufficient in itself to generate EMG myotonic activity, could account for some of the sensitivity to stretch and symptoms of stiffness. It also provides evidence for a general muscle membrane abnormality in this disorder. This conclusion is further supported by the raised creatine kinase activity found in all four members of the family.

Because of the mildness and non-progressive nature of the symptoms in the members of our family, it was decided that no treatment should be given. They have been followed up for a period of up to 12 years now and there has been no change in their clinical state.

1 Denny-Brown D, Pennybacker JB. Fibrillation and fasciculation in voluntary muscle. Brain 1938;61:311-34.

2 Salick AI, Pearson CM. Electrical silence of myoedema. Neurology 1967;17:899-901.

3 Torbergsen T. A family with dominant hereditary myotonia, muscular hypertrophy and increased muscular irritability, distinct from myotonia congenita Thomsen. Acta Neurol Scand 1975;51:225-32.

4 Ricker K, Moxley RT, Rohkamm R. Rippling muscle disease. Arch Neurol 1989;46:405-8.

5 Hylton D, Humphreys P, Keene D, Simpson C. A new syndrome of exercise-induced muscle rippling, muscle

6 Jusic A. Hereditary increased muscle mechanical irritability and progressive contracture with stretch-induced bility and progressive contracture with stretch-induced
electromyographic activity. Muscle Nerve 1989;12: electrom 103 .

7 Alberca R, Rafel E, Castilla JM, Gil-Peralta A. Increased mechanical muscle irritability syndrome. Acta Neurol Scand 1980;62:250-54.

8 Rao KR, Hafford JM, Grunnet $M$, Greenstein $M$. Electrically silent muscle contractions, myoedema and myopathy. Muscle Nerve 1986;9:663.

9 Bretag AH. Synthetic interstitial fluid for isolated mammalian tissue. Life Sci 1969;8:319-29.

10 Bretag AH, Dawe SR, Kerr DIB, Moskwa AG. Myotonia as a side-effect of diuretic action. $B r f$ Pharmacol 1980;71:467-71.

11 Kwiecinski H, Lehmann-Horn F, Rüdel R. The resting membrane parameters of human intercostal muscle at low, normal and high extracellular potassium. Muscle low, normal and high $1984 ; 7: 60-5$.

12 Adrian RH, Bryant $\mathrm{SH}$. On the repetitive discharge in myotonic muscle fibres. I Physiol (Lond) 1974;240: 505-15.

13 Nicholson GA, McLeod JG, Morgan G, et al. Variable distribution of serum creatine kinase reference values: Relationship to exercise activity. $\mathcal{F}$ Neurol Sci 1985;71: 233-45. 14 Jones MP, Parkes WE. Myoidema. Clin Sci 1955;14: 15 Taylor GF, Chhuttani PN. Myoidema. BMF 1949;ii:

16 Mizusawa H, Takagi A, Sugita H, Toyokura Y. Mounding phenomenon: an experimental study in vitro. Neurology 1983;33:90-3.

17 Mizusawa H, Takagi A, Nonaka I, Sugita H, Toyokura Y. Muscular abnormalities in experimental hypothyroidism of rats with special reference to the mounding phenomenon. Exp Neurol 1984;85:480-92. 